https://helda.helsinki.fi

Physical activity and peripheral artery disease : Two prospective cohort studies and a systematic review

\title{
Heikkila, Katriina
}

2019-07

Heikkila , K, Coughlin , P A , Pentti , J , Kivimäki , M \& Halonen , J 2019 , ' Physical activity and peripheral artery disease : Two prospective cohort studies and a systematic review ' , Atherosclerosis , vol. 286 , pp. 114-120 . https://doi.org/10.1016/j.atherosclerosis.2019.05.008

http://hdl.handle.net/10138/318021

https://doi.org/10.1016/j.atherosclerosis.2019.05.008

publishedVersion

Downloaded from Helda, University of Helsinki institutional repository.

This is an electronic reprint of the original article.

This reprint may differ from the original in pagination and typographic detail.

Please cite the original version. 


\title{
Physical activity and peripheral artery disease: Two prospective cohort studies and a systematic review
}

\author{
Katriina Heikkilä ${ }^{\mathrm{a}, \mathrm{b}, *}$, Patrick A. Coughlin ${ }^{\mathrm{c}}$, Jaana Pentti ${ }^{\mathrm{d}}$, Mika Kivimäki ${ }^{\mathrm{d}, \mathrm{e}}$, Jaana I. Halonen ${ }^{\mathrm{d}, \mathrm{f}}$ \\ ${ }^{a}$ Department of Health Services Research and Policy, London School of Hygiene and Tropical Medicine, London, UK \\ ${ }^{\mathrm{b}}$ Clinical Effectiveness Unit, the Royal College of Surgeons of England, London, UK \\ ${ }^{\mathrm{c}}$ Division of Vascular and Endovascular Surgery, Addenbrooke's Hospital, Cambridge University Hospital Trust, Cambridge, UK \\ ${ }^{\mathrm{d}}$ Department of Public Health, Faculty of Medicine, University of Helsinki, Finland \\ ${ }^{\mathrm{e}}$ Department of Epidemiology and Public Health, University College London, London, UK \\ ${ }_{\mathrm{f}}^{\mathrm{f}}$ Finnish Institute of Occupational Health, Kuopio, Finland
}

\section{H I G H L I G H T S}

- Physical activity is a modifiable risk factor for cardiovascular disease.

- Individuals with a diagnosis of peripheral artery disease (PAD) tend to be less physically active, regardless of whether activity was self-reported or measured objectively.

- The findings from the longitudinal studies suggest that more intense physical activity is associated with lower risk of developing PAD.

\section{A R T I C L E I N F O}

\section{Keywords:}

Peripheral artery disease

Intermittent claudication

Cardiovascular disease

Physical activity

Exercise

\begin{abstract}
A B S T R A C T
Background and aims: Physical activity is a modifiable risk factor for cardiovascular disease and an important therapy in individuals with intermittent claudication. However, its role in the development of peripheral artery disease (PAD) is unclear. We have examined the evidence of the association between physical activity and development of PAD.

Methods: We searched PubMed, EMBASE and CINAHL Plus in August 2018 for original studies of physical activity and PAD. Studies reporting prevalence or incidence of PAD by categories of physical activity (an amount of activity per unit of time) were included. In addition, we analysed unpublished individual-level data from two register-linked cohort studies, Finnish Public Sector Study $(n=63,924)$ and Whitehall II $(n=10,200)$. Due to heterogeneity in the assessment of physical activity and PAD, we provide a qualitative synthesis of the findings. Results: Evidence from 18 studies (15 cross-sectional/case-control and 7 prospective studies) of the association between physical activity and PAD in total of 152,188 participants, including 3971 PAD patients, suggests that individuals with a diagnosis or clinical findings of PAD were less physically active, regardless of whether activity was self-reported or measured using accelerometers. The findings from the longitudinal studies point to more intense physical activity being associated with lower odds of developing PAD; however, the study-specific findings lacked power to precisely estimate this relationship.

Conclusions: Individuals with PAD were less physically active than those without PAD. The longitudinal findings suggest that physical activity decreases the risk of PAD, although better powered studies are needed to confirm this.
\end{abstract}

\section{Introduction}

Peripheral artery disease (PAD) affects over 200 million people worldwide and it is the third most common cause of cardiovascular morbidity, after stroke and coronary heart disease [1]. With an increasing prevalence in both high- and low-income countries, PAD incurs considerable costs to patients and healthcare systems alike, in terms of decreased quality of life, loss of healthy life-years and medical

\footnotetext{
* Corresponding author. Department of Health Services Research and Policy, London School of Hygiene and Tropical Medicine, 15-17 Tavistock Place, London, WC1H 9SH, United Kingdom.

E-mail address: katriina.heikkila@lshtm.ac.uk (K. Heikkilä).
} 
and surgical treatment $[2,3]$. Despite the increasing burden of PAD, the evidence on risk factors specific to this manifestation of cardiovascular disease is scarce, and specific treatment targets are lacking [4].

At present the primary and secondary preventive treatment for PAD is identical to the prevention of coronary and carotid atherosclerotic disease, with reducing cholesterol, hypertension, smoking, and obesity as the main interventional targets [4]. Although different manifestations of cardiovascular disease share many risk factors, the magnitude of the risk incurred by specific exposures varies between disease outcomes. For example, smoking, hypertension and type- 2 diabetes have been consistently shown to be associated with an increased risk of coronary artery disease as well as PAD [1,5], but there is evidence that the effects of other risk factors may differ, e.g. with cholesterol being more strongly implicated in coronary heart disease and chronic inflammation in PAD [6]. Whilst addressing the coronary and carotid therapeutic targets is undoubtedly beneficial to patients at risk of PAD or living with this disease, more explicit evidence on the risk factors specific to peripheral artery atherosclerotic disease is needed to inform clinical practice and the development of guidelines aiming to reduce the incidence and improve prognosis of PAD at individual- and population-levels.

One important modifiable risk factor for cardiovascular disease is low level of physical activity [7]. Randomised controlled trials have shown that physical activity interventions, particularly supervised exercise programmes, are also effective secondary preventive measures in patients with intermittent claudication, as they improve patient-relevant outcomes, such as overall walking distance and speed as well as pain-free walking distance in this patient group [8,9]. However, the potential role of self-initiated physical activity in the development of PAD is unclear. A link between physical activity and the incidence of PAD would lend weight to the increasing role of physical activity and exercise as part of primary prevention strategies for atherosclerotic cardiovascular disease in general and PAD in particular. Here we have systematically reviewed and summarised the currently available evidence of association between self-initiated physical activity and PAD.

\section{Materials and methods}

\subsection{Systematic searches}

We searched PubMed, EMBASE and CINAHL Plus from the inception of each database up to 31 August 2018 for studies comparing physical activity levels in individuals with and without PAD. Briefly, we searched for original studies in humans, indexed with "physical activity" or "exercise" and "peripheral artery occlusive disease" or "peripheral artery disease" as keywords or Medical Subject Heading terms. No limits were set on study design or language of publication. Details of the search terms used are provided in Online Supplement.

Two reviewers (KH and $\mathrm{JIH}$ ) independently screened titles and abstracts, reviewed full-text articles, selected relevant ones for inclusion, and extracted data on participant characteristics, association estimates and their confidence intervals. Any discrepancies or disagreements in identifying studies or extracting data were solved by discussion with a third reviewer (PAC). Relevant version (for case-control, cross-sectional or cohort studies) of the Newcastle-Ottawa scale was used to assess the risk of bias in the included studies.

\subsection{Inclusion and exclusion criteria}

The key inclusion criterion was that the studies reported results on physical activity, as an amount of activity per unit of time, for individuals with and without PAD. Where more than one article described analyses of physical activity and PAD in the same dataset $[5,10]$, the more recently published or larger study was included in the summary of evidence [10].

Laboratory-based studies, case-studies, case series and reviews were excluded. We further excluded studies in which physical activity was not reported as an amount of activity per unit of time. To facilitate within- and between-studies comparisons, studies in which all participants had PAD or all participants were free of PAD during the studyperiod were excluded. As the focus of our review was to examine the associations between self-initiated physical activity and PAD, studies of physical activity interventions as a secondary preventative measure in individuals with PAD were excluded. This literature has been extensively reviewed previously $[8,9,11]$ and the findings are compared to those of the current review in the Discussion.

\subsection{Additional analyses on unpublished prospective individual-participant data}

In addition to the published studies, we investigated the association between physical activity and incident PAD using unpublished individual-participant data from two ongoing prospective cohort studies, Finnish Public Sector study (63,924 public sector employees, aged $17-69$ at baseline in 2000 or 2004) and Whitehall II (10,200 British Civil Service employees, aged $35-55$ at baseline in 1985-1989). All participants gave informed consent to take part and both study protocols conform to the ethical guidelines of the 1975 Declaration of Helsinki. The Finnish Public Sector Study was approved by the Helsinki and Uusimaa hospital district ethics committee. The Whitehall II study protocol was approved by the University College London Medical School committee on the ethics of human research.

Physical activity (hours per week) was self-reported at baseline in both studies. The participants' study records were linked to national hospitalisation datasets for information on episodes of hospital care, and the incidence of lower limb PAD during follow-up was ascertained from the hospitalisation data using International Classification of Diseases (ICD) versions 8, 9 and 10 codes indicating intermittent claudication or lower limb ischaemia, or the Office for Populations Censuses and Surveys (OPCS) version 4 code indicating a lower limb procedure relating to PAD (Supplementary Tables S1 and S2). We used Cox proportional hazards regression (with years since baseline as the time-scale) to examine the associations between physical activity at baseline and PAD during follow-up. Analyses were adjusted for age (years), sex, socioeconomic position and smoking (never, ex-, current). Details of the Finnish Public Sector study and Whitehall II, as well as their participants, are provided in the Supplementary Section 4 and Table S5.

\subsection{Evidence synthesis}

The assessment and definition of physical activity as well as the ascertainment of PAD varied between studies. For this reason we decided against pooling the study-specific estimates in meta-analyses and have provided a qualitative summary of the findings instead.

\section{Results}

\subsection{Systematic search}

The results of the systematic searches are detailed in Fig. 1. Briefly, we screened 4436 abstracts and selected 35 potentially relevant articles for full-text review. Of these, 18 articles were excluded. The main reasons for exclusion were irrelevant exposure (other than an amount of physical activity per unit of time) or irrelevant outcome (e.g. CVD other than PAD). After all exclusions, and adding one eligible article from the citing reference search, 18 articles reporting on the association of physical activity with prevalent or incident PAD were included in the review [10,12-28]. 


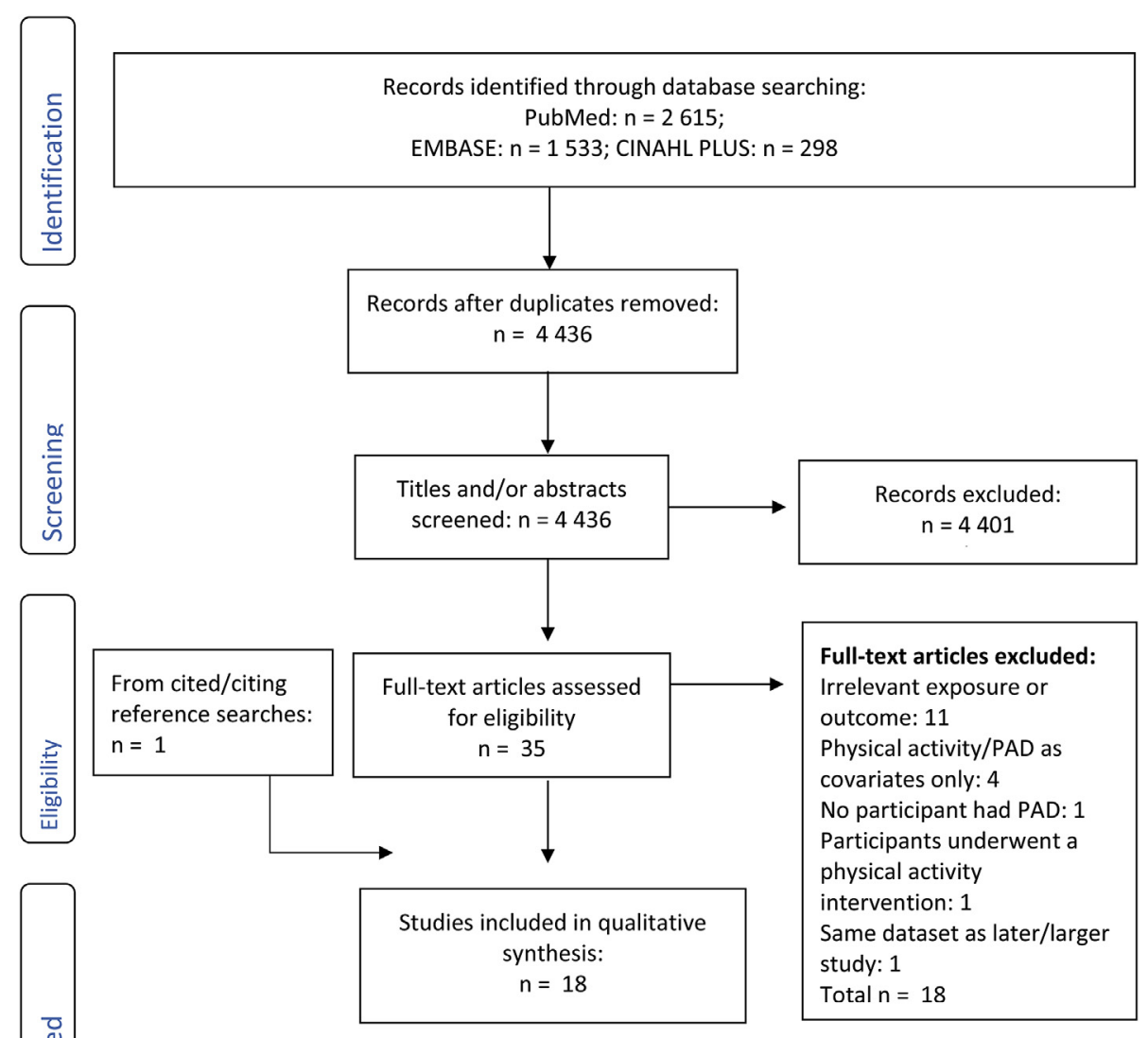

Fig. 1. Systematic search flow diagram.

\subsection{Study characteristics}

A large majority of the studies we reviewed $(n=15)$ had a crosssectional or case-control design [10,12-14,16-20,22-26,28] (Table 1 and Supplementary Table S3). Longitudinal analyses were conducted in only five studies $[15,16,18,21,27]$ (Table 2 and Supplementary Table S4). Two articles contained both cross-sectional and longitudinal findings $[16,18]$.

In most studies, self-initiated physical activity was self-reported by the participants $[10,12-16,18,21,22,25,27,28]$, whereas objectively measured physical activity data, using accelerometers, were collected in seven studies [17,19,20,23-26]. The most common method for identifying patients with PAD was the measurement of ankle-brachial index (ABI: the ratio of blood pressure in the brachial artery and the dorsalis pedis and posterior tibial arteries). PAD was typically defined as having $\mathrm{ABI}<0.9[10,14-17,22-24,27,28]$, though a threshold of either below 0.9 or above 1.4 (abnormal ABI) was also used in two sets of analyses [20,22]. A combination of having an ABI below the 0.9 threshold and a clinical diagnosis of PAD was used in three studies $[12,13,25]$ and one study ascertained incident PAD on the basis of at least one of the following: $\mathrm{ABI}$ lower than 0.9 at rest, clinical evidence of arterial occlusive disease, or an endovascular or open surgical revascularisation or amputation [21]. In one study PAD was defined based on the Edinburgh Claudication Questionnaire or having undergone a PAD-related procedure [18].

Most studies presented results that were adjusted for a number of cardiovascular disease risk factors and other covariates that could confound any observed association between physical activity levels and PAD $[10,13,16,18-21,24,25]$. However, in one study the findings were adjusted for age [14] and five studies presented only unadjusted results
$[12,16,22,23,28]$.

\subsection{Findings from cross-sectional and case-control studies}

In most cross-sectional and case-control studies individuals with PAD undertook less self-initiated physical activity and exercised less strenuously than those without PAD [10,13,14,16-20,23-26]. The direction of this association was the same in unadjusted analyses $[16,23,26]$, those adjusted for age alone [14], analyses with adjustment for multiple CVD risk factors and potential determinants of physical activity levels [10,13,17-20,24,25] as well as in the investigations where all participants had chronic kidney disease, diabetes, coronary artery disease or heart failure $[13,19,20]$. By contrast, in two studies the investigators reported higher prevalence or odds of PAD in moderately active participants compared to sedentary participants [12,22]. In addition, one article reported no association between $\mathrm{ABI}$ and quantiles of physical activity [28].

A large majority of cross-sectional and case-control studies were based on relatively small numbers of PAD patients (n with PAD $<200$, Supplementary Table S3), although two larger studies from Australia and the United States had 638 and 754 PAD cases, respectively [13,18]. Also, as expected of PAD patients, many study participants had other, comorbid diseases (e.g. type-2 diabetes), but the detail to which these were reported varied. In three studies all participants had a chronic disease: populations with chronic kidney disease [13], diabetes [20] or coronary artery disease or chronic heart failure [19] were examined.

An important barrier to physical activity in PAD patients is intermittent claudication, but the presence or absence of this symptom was incompletely reported in the studies we reviewed. In the only study focusing on truly asymptomatic patients, the investigators reported 
Table 1

Summary of findings from cross-sectional and case-control studies of physical activity and peripheral artery disease (PAD).

\begin{tabular}{|c|c|}
\hline Reference & Main findings \\
\hline \multirow[t]{3}{*}{ Berard [12] } & OR (95\% CI) for PAD: \\
\hline & No physical activity (ref. cat.) \\
\hline & Some physical activity: $2.7(1.4-5.4)$ \\
\hline Chen [13] & OR (95\% CI) for PAD, per 1 SD change in METs/week (146 METs): $0.87(0.78-0.97)$ \\
\hline \multirow{4}{*}{ Collins [14] } & OR $(95 \% \mathrm{CI})$ for PAD: \\
\hline & Reference category: ideal activity level ( $\geq 150 \mathrm{~min} /$ week of moderate and/or vigorous activity) \\
\hline & Poor (no physical activity): $1.35(1.00-1.82)$ \\
\hline & Intermediate (1-149 $\mathrm{min} / \mathrm{wk}$ moderate and/or vigorous activity): $0.98(0.69-1.38)$ \\
\hline \multirow[t]{4}{*}{ Engström [16] } & Mean (SD) ABI: \\
\hline & Inactive: $0.89(0.21)$ \\
\hline & Light activity $\geq 4$ hrs/week: $1.01(0.13)$ \\
\hline & Moderate/heavy activity $\geq 2-3 \mathrm{~h} /$ week or competitive sport several times a week: $1.05(0.11)$ \\
\hline \multirow[t]{2}{*}{ Kulinski [17] } & OR $(95 \% \mathrm{CI})$ for PAD, per 1 SD (13 min) increase in daily exercise time: $0.71(0.57-0.89)$ \\
\hline & Findings based on objectively measured physical activity. \\
\hline \multirow[t]{3}{*}{ Lakshmanan [18] } & OR $(95 \% \mathrm{CI})$ for PAD: \\
\hline & Exercise $<3 \mathrm{~h} /$ week: ref. cat. \\
\hline & Exercise $\geq 3 \mathrm{~h}$ /week: $0.78(0.66-0.93)$ \\
\hline \multirow[t]{2}{*}{ Loprinzi 2016 [19] } & OR $(95 \% \mathrm{CI})$ for PAD, per $60 \mathrm{~min} /$ day increase in physical activity: $0.81(0.67-0.99)$ \\
\hline & Findings based on objectively measured physical activity. \\
\hline Loprinzi 2014 [20] & OR for PAD, per minute of moderate/vigorous physical activity (activity count $\geq 2020 / \mathrm{min}$ ): $0.77(0.62-0.96)$ \\
\hline Maggio [22] & Participants with PAD were more active than those without PAD. \\
\hline \multirow[t]{2}{*}{ McGrae McDermott [23] } & Mean (SD) activity units: ABI < 0.9: 783.8 (426.2); ABI 0.9-1-5: 1109 (640.1); $p<0.001$ \\
\hline & Findings based on objectively measured physical activity. \\
\hline \multirow[t]{6}{*}{ Parsons [24] } & OR for $\mathrm{ABI}<0.9(95 \% \mathrm{CI})$ : \\
\hline & ...per 10,000 vertical counts/day: $0.92(0.88-0.96)$ \\
\hline & ...per 1000 steps/day: $0.74(0.65-0.86)$ \\
\hline & ...per 30min LPA/day: $0.85(0.74-0.97)$ \\
\hline & ...per 10min MVPA/day: $0.79(0.70-0.90)$ \\
\hline & Findings based on objectively measured physical activity. \\
\hline \multirow[t]{4}{*}{ Ruiz Comellas [10] } & OR $(95 \% \mathrm{CI})$ for PAD: \\
\hline & Walking, < $0.5 \mathrm{~h} /$ day: ref. cat. \\
\hline & Walking, 0.5-1 h/day: $0.78(0.49-1.25)$ \\
\hline & Walking, > 1hr/day: $0.55(0.35-0.84)$ \\
\hline \multirow[t]{5}{*}{ Sieminski [25] } & Mean (SD) kcal/day, measured by Caltrac accelerometer: PAD: 384 (212); No PAD: 563 (363); $p<0.001$ \\
\hline & Mean (SD) steps/day, measured by pedometer: PAD: 5163 (3273); No PAD: 9123 (5162); $p<0.001$ \\
\hline & Mean (SD) kcal/day, measured by Minnesota LTPA: PAD: 149 (161); No PAD: 226 (251); $p<0.001$ \\
\hline & Mean (SD) activity code, measured by JCS PAS: PAD: 1.5 (1.0); No PAD: 2.7 (1.9); $p<0.001$ \\
\hline & Findings based on objectively measured physical activity. \\
\hline \multirow[t]{4}{*}{ Siscovick [26] } & N (\%) with PAD, by physical activity category: \\
\hline & Men: Low intensity: 13 (18.3); Medium intensity: 42 (5.5); High intensity, MET > 6:7 (3.7); $p=0.01$ \\
\hline & Women: Low intensity: 19 (10.0); Medium intensity: 50 (5.7); High intensity, MET > 6: 5 (2.8); $p=0.02$ \\
\hline & Findings based on objectively measured physical activity. \\
\hline \multirow[t]{2}{*}{ Wilson [28] } & Mean (SD) ABI: \\
\hline & No LRA: 0.94 (0.21); LRA Quantile 2: 1.02 (0.18); LRA Q3: 1.03 (0.18); LRA Q4: 1.02 (0.18); LRA Q5: $1.02(0.17) ; p<0.001$ \\
\hline
\end{tabular}

ABI: ankle-brachial index; OR: odds ratio; CI: confidence interval; HDL: high density lipoprotein cholesterol; GFR: glomerular filtration rate; BMI: body mass index; CRP: C-reactive protein; HgbA1C: glycated haemoglobin; CVD: cardiovascular disease; LPA: light physical activity; MVPA: moderate/vigorous physical activity; LTPA: leisure-time physical activity; kcal: kilocalories; MET: metabolic equivalent; SBP: systolic blood pressure; WHR: waist:hip ratio; LRA: lifetime recreational activity; Q: quantile.

lower mean $\mathrm{ABI}$ values in men who were inactive and higher values in those who undertook at least $4 \mathrm{~h}$ of light activity per week [16]. In the majority of the studies in which PAD patients' physical activity levels were lower than those of comparison groups', PAD was defined as an $\mathrm{ABI} \leq 0.9$, and it is possible that the low ABI group included patients with other diseases characterised by low ABI [10,14,17,19,20,23-26]. It is therefore possible that the observed co-occurrence of low levels of physical activity and PAD in these studies represents diseases other than PAD (e.g. chronic obstructive pulmonary disease, arthritis or angina pectoris) impairing the patients' ability to undertake physical activity.

Taken together, the findings from the cross-sectional and casecontrol studies suggest that men and women with PAD are less physically active than those free of this disease. However, in two studies PAD patients were more active than the comparison group [12,22]. These contradicting findings may be residual confounding, as the latter analyses lacked adjustment for potential confounders. Another possibility is that these observations represent individuals with early PAD (undiagnosed before the onset of the study) using physical activity to alleviate the symptoms of the disease.

\subsection{Findings from longitudinal analyses}

The results from all seven longitudinal analyses suggested that the more physical activity the participants reported at baseline, the lower their odds of developing PAD during follow-up were [15,16,18,21,27] (Whitehall II and Finnish Public Sector study, Table 2 and Supplementary Table S4). However, the $95 \%$ confidence intervals for all bar two of the point estimates were wide and crossed the null-value. In the Spanish PREDIMED study (number with incident PAD: 87) the HR for PAD over a median of 4.8 years of follow-up was 0.45 (95\% CI: 0.27 to 0.76 ) in individuals reporting leisure-time physical activity of $\geq 500$ MET minutes/week, compared to those whose physical activity levels were lower [21]. In the Finnish Public Sector study (n incident PAD: 106) the HR for PAD over up to 11 years of follow-up was 0.53 (95\% CI: 0.32 to 0.87 ) among those reporting 30 MET-hours of physical activity per week (Table 2).

One explanation for the lack of clear evidence for an association between physical activity and incident PAD is that the longitudinal studies were underpowered to detect an association between levels of physical activity and PAD. Although many were based on relatively 
Table 2

Summary of findings from longitudinal studies of physical activity and peripheral artery disease (PAD).

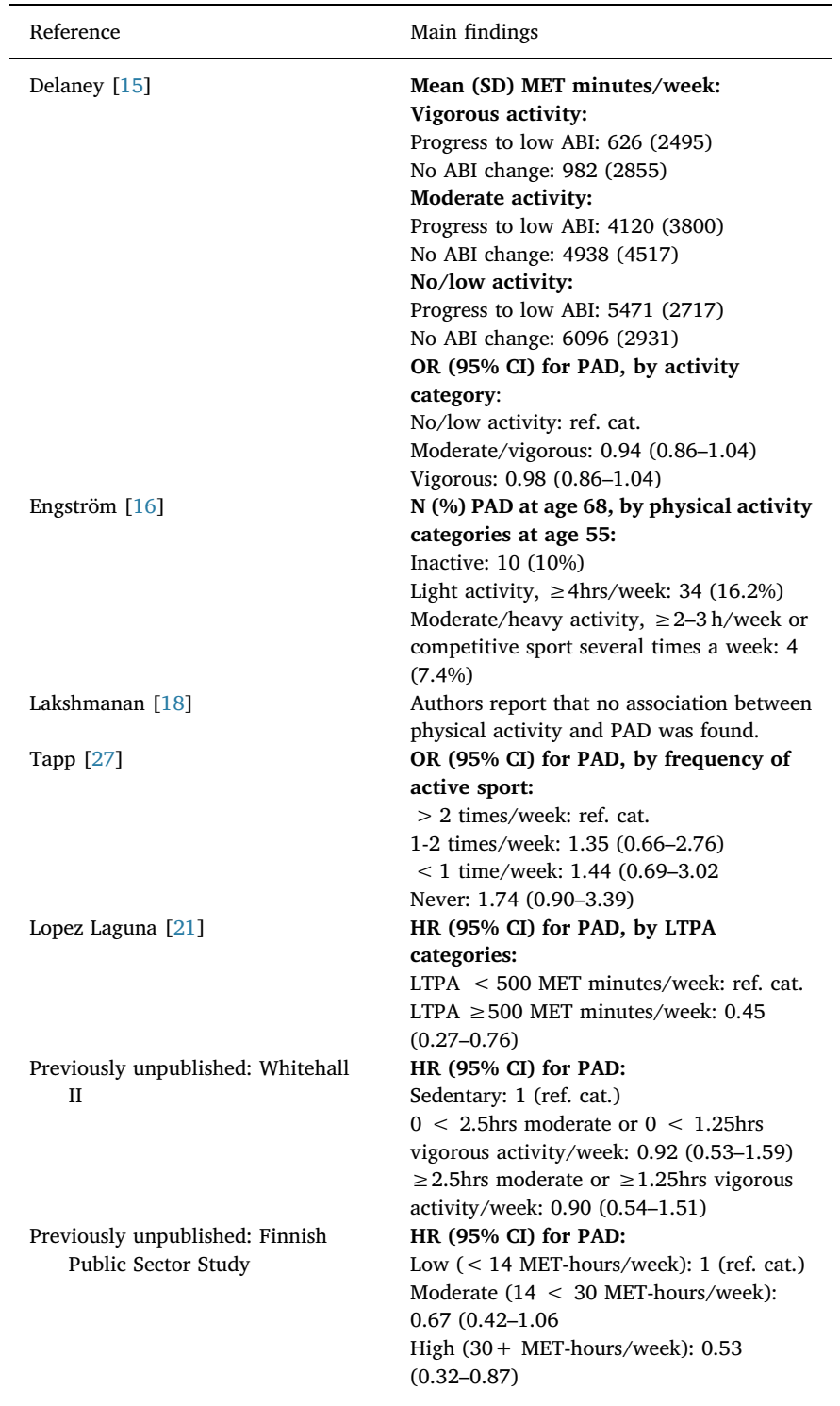

ABI: ankle-brachial index; OR: odds ratio; $\mathrm{CI}$ : confidence interval; SD: standard deviation; MET: metabolic equivalent; SBP: systolic blood pressure; CHD: coronary heart disease; HRT: hormone replacement therapy.

large overall sample sizes, the number of incident PAD cases ranged from 48 to 193 (Supplementary Table S4). Another possible reason for the point-estimates indicating an association but the 95\% confidence intervals failing to support a trend is that although the investigators made an effort to exclude from the analyses participants who had PAD at baseline, some individuals may have had a sub-clinical PAD at the time physical activity was assessed (e.g. normal resting ABI measurements, but some PAD with good collateralisation), and thus reverse causality (from early symptoms of PAD causing an individual to be less physically active) could have diluted the observed associations. Similarly to the cross-sectional studies, the longitudinal studies we reviewed provided limited and heterogeneous information on the patients' symptom status: PAD was defined based on low ABI alone [15], intermittent claudication or a procedure for PAD [18], low ABI and lack of symptoms [16], a combination of low ABI and history of revascularisation [27] and hospital records of PAD diagnosis or lower limb revascularisation (Finnish Public Sector study and Whitehall II). Only one of the longitudinal studies explicitly reported that individuals with PAD at baseline were excluded from the analyses [21]. In our analyses in Whitehall II, no data were available on the participants' PAD-status before the baseline; however, as the first incident PAD event took place seven years post-baseline, it seems unlikely that a large number of prevalent PAD cases would have been included in our analyses. In the Finnish Public Sector study individuals with a hospital record of PAD at or before baseline were excluded from the analyses.

\section{Discussion}

\subsection{Summary of findings}

Physical activity is an important therapeutic target in treating patients with intermittent claudication (an early manifestation of PAD), and encouraging results from clinical trials point to the impact of supervised exercise programmes in improving functional capacity in this group of patients $[8,9,11]$. The findings of our systematic review provide some indication that self-initiated physical activity is associated with a decreased risk of PAD. However, all the evidence presented here comes from European high-income countries with universal healthcare systems, and it is possible that the associations between physical activity and PAD are different in low-income settings and in contexts of limited healthcare access.

\subsection{Risk of bias and potential sources for heterogeneity}

We are not aware of previous data syntheses of the association between self-initiated physical activity and the incidence of PAD, which precludes comparison to other reviews. A large majority of the research in this area is based on case-control or cross-sectional studies, which can only provide evidence on the co-occurrence of physical activity or exercise intensity and PAD. Such findings provide limited information on the role of physical activity in the development of PAD, as the presence of the disease will likely impact on participants' propensity to undertake physical activity, explaining the observations of PAD patients having lower levels of physical activity than PAD-free individuals.

The risk of bias in the studies we reviewed varied: many studies were generally well-conducted, using appropriate measures of physical activity and PAD outcomes, with the investigators reporting estimates adjusted for a number of potentially confounding CVD risk factors and determinants of physical activity. However, some study reports contained unadjusted estimates. The main limitation in the evidence-base is that a large majority of studies included in our review were based on small numbers of PAD patients and thus lacked statistical power to accurately estimate the association between physical activity levels and concurrent or incident PAD.

Due to differences in the definitions of physical activity and ascertainment of PAD, individual study findings were not pooled in metaanalyses. However, our qualitative summary of the results indicates that individuals with PAD tend to be less physically active and that larger studies, with uniform measurement of physical activity and PAD outcomes, would be needed to ascertain whether physical activity has a role in the development of this disease. One way to collect objectively measured data on duration and intensity of physical activity would be to use accelerometers now widely available as smart phone apps. As demonstrated by research in Parkinson's disease, mobile phone accelerometers enable the collection of uniform data on physical activity in older people [29]. This approach could be extended to investigating physical activity in individuals with PAD or those at risk of developing this disease. Also, PAD affects legs and thus limits the patients' ability to walk, but it does not tend to affect cycling and many patients are able to swim. Future studies would do well to distinguish between different types of physical activity in order to determine whether the degree to which association of low levels of physical activity with PAD reflects walking impairment alone.

The overall findings of our systematic review reflect those of 
randomised controlled trials of exercise interventions among patients with intermittent claudication. High quality evidence shows that exercise programmes in general improve pain-free and maximum walking distance in people with intermittent claudication [9], and that supervised exercise therapies are more effective at this than home-based exercise interventions or walking advice, showing benefits that translate to an up to $210 \mathrm{~m}$ ' increase in maximum walking distance after three months of therapy [8]. However, the trials provide no clear evidence of exercise improving ABI or flow-mediated dilatation, or having an impact on the risk of amputation or death in patients with intermittent claudication, suggesting that although exercise is important in treatment of intermittent claudication, is does not necessarily slow down or stop disease progression $[9,11]$. Thus, although our review provides some indication that higher levels of self-initiated physical activity may be associated with a lower incidence of PAD and good quality evidence shows that exercise interventions improve functional capacity and quality of life in those who already have the disease, it seems that the benefits of physical activity do not directly translate into an improved vascular function or lower risk of peripheral artery atherosclerotic disease.

In conclusion, individuals with PAD tend to be less physically active than those without the disease. The longitudinal findings suggest that physical activity decreases the risk of PAD, although better powered studies are needed to confirm this.

\section{Conflict of interest}

The authors declared they do not have anything to disclose regarding conflict of interest with respect to this manuscript.

\section{Financial support}

Whitehall II study is supported by grants from the UK Medical Research Council (K013351, R024227), the US National Institutes on Aging (R01AG056477) and the British Heart Foundation (32334). The Finnish Public Sector study is supported by the Finnish Institute of Occupational Health.

\section{Author contributions}

$\mathrm{KH}$ and JIH designed the study, with input for PAC. KH, JIH, PAC, JP and MK participated in acquisition, analysis and interpretation of data. $\mathrm{KH}$ wrote the first draft of the article and all authors participated in revising it critically for important intellectual content. All authors approved the version to be published.

\section{Ethical approval}

The Finnish Public Sector Study was approved by the Helsinki and Uusimaa hospital district ethics committee. The Whitehall II study protocol was approved by the University College London Medical School committee on the ethics of human research. All procedures performed in these studies were in accordance with the ethical standards of the institutional and/or national research committee and with the 1964 Helsinki declaration and its later amendments or comparable ethical standards. Informed consent was obtained from all individual participants included in The Finnish Public Sector Study and Whitehall II.

\section{Acknowledgements}

The authors thank all of the participating civil service departments in Whitehall II study and their welfare, personnel, and establishment officers; the British Occupational Health and Safety Agency; the British Council of Civil Service Unions; all participating civil servants in the Whitehall II study; and all members of the Whitehall II study team. The
Whitehall II Study team comprises research scientists, statisticians, study coordinators, nurses, data managers, administrative assistants and data entry staff, who make the study possible. The authors also thank all the participants of the Finnish Public Sector study and the study team at the Finnish Institute of Occupational Health.

\section{Appendix A. Supplementary data}

Supplementary data to this article can be found online at https:// doi.org/10.1016/j.atherosclerosis.2019.05.008.

\section{References}

[1] F.G. Fowkes, D. Rudan, I. Rudan, V. Aboyans, J.O. Denenberg, M.M. McDermott, P.E. Norman, U.K. Sampson, L.J. Williams, G.A. Mensah, et al., Comparison of global estimates of prevalence and risk factors for peripheral artery disease in 2000 and 2010: a systematic review and analysis, Lancet 382 (9901) (2013) 1329-1340.

[2] E.M. Mahoney, K. Wang, H.H. Keo, S. Duval, K.G. Smolderen, D.J. Cohen, G. Steg, D.L. Bhatt, A.T. Hirsch, Reduction of Atherothrombosis for Continued Health Registry I: vascular hospitalization rates and costs in patients with peripheral artery disease in the United States, Circ Cardiovasc Qual Outcomes 3 (6) (2010) 642-651.

[3] A.T. Hirsch, L. Hartman, R.J. Town, B.A. Virnig, National health care costs of peripheral arterial disease in the Medicare population, Vasc. Med. 13 (3) (2008) 209-215.

[4] M.F. Piepoli, A.W. Hoes, S. Agewall, C. Albus, C. Brotons, A.L. Catapano, M.T. Cooney, U. Corra, B. Cosyns, C. Deaton, et al., European guidelines on cardiovascular disease prevention in clinical practice: the sixth joint task force of the european society of cardiology and other societies on cardiovascular disease prevention in clinical practice (constituted by representatives of 10 societies and by invited experts)Developed with the special contribution of the european association for cardiovascular prevention \& rehabilitation (EACPR), Eur. Heart J. 37 (29) (2016) 2315-2381 2016.

[5] M.T. Alzamora, R. Fores, J.M. Baena-Diez, G. Pera, P. Toran, M. Sorribes, M. Vicheto, M.D. Reina, A. Sancho, C. Albaladejo, et al., The peripheral arterial disease study (PERART/ARTPER): prevalence and risk factors in the general population, BMC Public Health 10 (2010) 38.

[6] H. Tunstall-Pedoe, S.A.E. Peters, M. Woodward, A.D. Struthers, J.J.F. Belch, Twenty-year predictors of peripheral arterial disease compared with coronary heart disease in the scottish heart health extended cohort (SHHEC), J. Am. Heart Assoc. 6 (9) (2017).

[7] A. Wahid, N. Manek, M. Nichols, P. Kelly, C. Foster, P. Webster, A. Kaur, C.F. Smith, E. Wilkins, M. Rayner, et al., Quantifying the association between physical activity and cardiovascular disease and diabetes: a systematic review and meta-analysis, J. Am. Heart Assoc. 5 (9) (2016).

[8] D. Hageman, H.J. Fokkenrood, L.N. Gommans, M.M. van den Houten, J.A. Teijink, Supervised exercise therapy versus home-based exercise therapy versus walking advice for intermittent claudication, Cochrane Database Syst. Rev. 4 (2018) CD005263.

[9] R. Lane, A. Harwood, L. Watson, G.C. Leng, Exercise for intermittent claudication, Cochrane Database Syst. Rev. 12 (2017) CD000990.

[10] A. Ruiz Comellas, G. Pera, J.M. Baena Diez, A. Heras, M.T. Alzamora Sas, R. Fores Raurell, P. Toran Monserrat, X. Mundet Tuduri, The relationship between physical activity in leasure time and the ankle-brachial index in a general Spanish population: the ARTPER study. [Spanish], Med. Clínica 145 (10) (2015) 419-426.

[11] B.J. Parmenter, G. Dieberg, N.A. Smart, Exercise training for management of peripheral arterial disease: a systematic review and meta-analysis, Sports Med. 45 (2) (2015) 231-244.

[12] A.M. Berard, A. Bedel, R. Le Trequesser, G. Freyburger, A. Nurden, S. Colomer, V. Guerin, M.C. Vergnes, F. Becker, G. Camelot, et al., Novel risk factors for premature peripheral arterial occlusive disease in non-diabetic patients: a case-control study, PLoS One 8 (3) (2013) e37882 (no pagination).

[13] J. Chen, I.E.R. Mohler, D. Xie, M.G. Shlipak, R.R. Townsend, L.J. Appel, D.S. Raj, A.O. Ojo, M.J. Schreiber, L.F. Strauss, et al., Risk factors for peripheral arterial disease among patients with chronic kidney disease, Am. J. Cardiol. 110 (1) (2012) $136-141$.

[14] T.C. Collins, D.P. Slovut, R. Newton, W.D. Johnson, S. Larrivee, J. Patterson, J.A. Johnston, A. Correa, Ideal cardiovascular health and peripheral artery disease in african Americans: results from the jackson heart study, Prev. Med. Rep. 7 (2017) 20-25.

[15] J.A. Delaney, N.E. Jensky, M.H. Criqui, M.C. Whitt-Glover, J.A. Lima, M.A. Allison, The association between physical activity and both incident coronary artery calcification and ankle brachial index progression: the multi-ethnic study of atherosclerosis, Atherosclerosis 230 (2) (2013) 278-283.

[16] G. Engstrom, M. Ogren, B. Hedblad, P. Wollmer, L. Janzon, Asymptomatic leg atherosclerosis is reduced by regular physical activity. Longitudinal results from the cohort "men born in 1914, Eur. J. Vasc. Endovasc. Surg. 21 (6) (2001) 502-507.

[17] J.P. Kulinski, M. Sanghavi, C.R. Ayers, S.R. Das, S. Banerjee, J.D. Berry, T. Addo, J.A. De Lemos, D.J. Kumbhani, Association between low ankle-brachial index and accelerometer-derived sedentary and exercise time in the asymptomatic general population, Vasc. Med. 20 (4) (2015) 332-338.

[18] R. Lakshmanan, Z. Hyde, K. Jamrozik, G.J. Hankey, P.E. Norman, Population-based observational study of claudication in older men: the Health in Men Study, Med. J. 
Aust. 192 (11) (2010) 641-645.

[19] P.D. Loprinzi, Physical activity and peripheral arterial disease among patients with coronary artery disease or congestive heart failure, Int. J. Cardiol. 207 (2016) 110-111.

[20] P.D. Loprinzi, K. Abbott, Association of diabetic peripheral arterial disease and objectively-measured physical activity: NHANES 2003-2004, J. Diabetes Metab. Disord. 13 (1) (2014) (no pagination)(63).

[21] N. Lopez-Laguna, M.A. Martinez-Gonzalez, E. Toledo, N. Babio, J.V. Sorli, E. Ros, M.A. Munoz, R. Estruch, J. Lapetra, C. Munoz-Bravo, et al., Risk of peripheral artery disease according to a healthy lifestyle score: the PREDIMED study, Atherosclerosis 275 (2018) 133-140.

[22] M. Maggio, C. Cattabiani, F. Lauretani, A. Artoni, S. Bandinelli, G. Schiavi, A. Vignali, R. Volpi, G. Ceresini, G. Lippi, et al., The relationship between sex hormones, sex hormone binding globulin and peripheral artery disease in older persons, Atherosclerosis 225 (2) (2012) 469-474.

[23] M.M. McDermott, P. Greenland, L. Ferrucci, M.H. Criqui, K. Liu, L. Sharma, C. Chan, L. Celic, A. Priyanath, J.M. Guralnik, Lower extremity performance is associated with daily life physical activity in individuals with and without peripheral arterial disease, J. Am. Geriatr. Soc. 50 (2) (2002) 247-255.
[24] T.J. Parsons, C. Sartini, E.A. Ellins, J.P. Halcox, K.E. Smith, S. Ash, L.T. Lennon, S.G. Wannamethee, I.M. Lee, P.H. Whincup, et al., Objectively measured physical activity and sedentary behaviour and ankle brachial index: cross-sectional and longitudinal associations in older men, Atherosclerosis 247 (2016) 28-34.

[25] D.J. Sieminski, A.W. Gardner, The relationship between free-living daily physical activity and the severity of peripheral arterial occlusive disease, Vasc. Med. 2 (4) (1997) 286-291.

[26] D.S. Siscovick, L. Fried, M. Mittelmark, G. Rutan, D. Bild, D.H. O'Leary, Exercise intensity and subclinical cardiovascular disease in the elderly. The Cardiovascular Health Study, Am. J. Epidemiol. 145 (11) (1997) 977-986.

[27] R.J. Tapp, B. Balkau, J.E. Shaw, P. Valensi, M. Cailleau, E. Eschwege, D.S. Group, Association of glucose metabolism, smoking and cardiovascular risk factors with incident peripheral arterial disease: the DESIR study, Atherosclerosis 190 (1) (2007) 84-89.

[28] A.M. Wilson, A.H. Sadrzadeh-Rafie, J. Myers, T. Assimes, K.T. Nead, M. Higgins, A. Gabriel, J. Olin, J.P. Cooke, Low lifetime recreational activity is a risk factor for peripheral arterial disease, J. Vasc. Surg. 54 (2) (2011) 427-432.

[29] M. Linares-del Rey, L. Vela-Desojo, R. Cano-de la Cuerda, Mobile phone applications in Parkinson's disease: a systematic review, Neurologia 34 (1) (2019) 38-54. 